\title{
Hyperbolic Method for Dispersive PDEs: Same High- Order of Accuracy for Solution, Gradient, and Hessian
}

\author{
Alireza Mazaheri* \\ NASA Langley Research Center, Hampton, VA 23681 \\ Mario Ricchiuto ${ }^{\dagger}$ \\ INRIA Bordeaux Sud-Ouest and Institut de Mathématiques de Bordeaux, 33405 Talence Cedex, France \\ Hiroaki Nishikawa ${ }^{\ddagger}$ \\ National Institute of Aerospace, Hampton, VA 23666
}

\begin{abstract}
In this paper, we introduce a new hyperbolic first-order system for general dispersive partial differential equations (PDEs). We then extend the proposed system to general advection-diffusion-dispersion PDEs. We apply the fourth-order RD scheme of Ref. 1 to the proposed hyperbolic system, and solve time-dependent dispersive equations, including the classical two-soliton $\mathrm{KdV}$ and a dispersive shock case. We demonstrate that the predicted results, including the gradient and Hessian (second derivative), are in a very good agreement with the exact solutions. We then show that the RD scheme applied to the proposed system accurately captures dispersive shocks without numerical oscillations. We also verify that the solution, gradient and Hessian are predicted with equal order of accuracy.
\end{abstract}

\section{Introduction}

Dispersion effects play a fundamental role in many applications involving hydrodynamics. At the large scale, the flow is dominated by advection, while the dissipative effects are more important at the microscopic level. At the mesoscopic level (the intermediate level) the dispersive effects become important, as it is the case, for example, in nonlinear optics, electromagnetism, quantum mechanics ${ }^{2-4}$ relativity and Bose-Einstein condensates, ${ }^{5,6}$ atmospheric, coastal, and fluvial hydrodynamics, ${ }^{7-11}$ magma, highly viscous fluids and/or capillary effects. ${ }^{12-14}$ The main physical effects associated with dispersion are the appearance of dispersive (or undulating) shocks, and the existence of smooth traveling solitary waves, which may produce complex interactions with one another. These systems, independent of the physical nature of the involved medium, admit a mesoscale hydrodynamic model, which consists of a set of Partial Differential Equations (PDEs). These PDEs are mostly regularizations of hyperbolic models that, in one-dimension, may be written as

$$
\partial_{t} u+\partial_{x} F(u)=\mathcal{D}
$$

where, in a classical sense (endowed with an entropy pair, with a diagonalizable flux Jacobian $F^{\prime}(u)$, etc.), the left hand side defines a hyperbolic model. Depending on the application and the physical hypotheses made, the regularization on the right hand side may take different forms, and have a dissipative and/or dispersive character (see e.g., Refs. 13,15-19). Therefore, term $\mathcal{D}$, may be a combination of the following forms:

(a) dissipative regularization $\mathcal{D}=\nu \partial_{x x} u$,

(b) dispersive regularization with time derivative $\mathcal{D}=\epsilon \partial_{x x t} u$,

\footnotetext{
*Research Aerospace Engineer, Aerothermodynamics Branch, M/S 408A, alireza.mazaheri@nasa.gov

${ }^{\dagger}$ Group Leader at INRIA Sud-Ouest, mario.ricchiuto@inria.fr

¥Associate Research Fellow, hiro@nianet.org
} 
(c) fully dispersive regularization $\mathcal{D}=\epsilon \partial_{x x x} u$.

The form (a) is a classical viscous regularization, while form (b) can be recast as a first-order evolutionary PDE with an embedded steady state second-order time independent elliptic problem; i.e.,

$$
\partial_{t} w=-\partial_{x} f, \quad-\partial_{x x} u+u=w .
$$

We previously showed e.g., in Refs. 1,20,21 how one can construct very accurate numerical approximations of problems of form (a) and the elliptic form of (b), by first reformulating the system of PDEs as a first-order hyperbolic system. The schemes proposed with the hyperbolic formulation of PDE systems are upwind and highly accurate for both the solution $u$ and its gradient $u_{x}$, and have a natural potential for extension on arbitrary unstructured meshes as illustrated in Refs. 22, 23 .

The presence of a third-order derivative term in form (c), however, introduces discretization difficulties, which are often related to the understanding of the type of stencil that is required to approximate these high-order derivative terms, the stability of the method used, and the imposition of boundary conditions. In Refs. 24 and 17, possible solutions to some of these issues are proposed, where the authors showed very good results with a non-hyperbolic first-order system reformulation of a PDE and careful discretization of the fluxes. The hyperbolic reformulation of dispersive PDEs similar to the one initially proposed for diffusion in Ref. 20 alleviate the above mentioned issues. However, it is shown and proved in Ref. 25 that the hyperbolic formulation of a dispersive PDE in the form given in Ref. 20 is not possible. Thus, we are motivated to introduce an alternative hyperbolic formulation that is carefully designed for general dispersive PDEs that are relevant to, for example, quantum mechanics, relativistic hydrodynamics, and coastal engineering applications, such as the Korteweg-de Vries (KdV) equation. ${ }^{15,18,24}$

In this work, we first present a first-order system for a pure dispersion equation, and show how the proposed formulation can be made hyperbolic. As an intermediate generalization, we then show that an advective-dispersive PDE (such as the classical KdV) can also be made fully-hyperbolic as well. The fully-hyperbolic advection-dispersion system could be useful in imposing a characteristics boundary condition. A practical extension of the proposed hyperbolic dispersion system for general advective-diffusivedispersive PDEs follows next. We then present some numerical examples by applying the high-order residualdistribution (RD) scheme of Ref. 1 to the proposed system, and solving general dispersive PDEs, including the classical KdV equation, on randomly distributed nodes. We verify the order of accuracy of the scheme for both the solution, the gradient, and the Hessian (second derivative) with the use of method of manufactured solution, and show that the RD scheme applied to the proposed hyperbolic system produces accurate solution, gradient and Hessian with equal order of accuracy. The ability to obtain accurate gradient and Hessian are very important as they are used in many hydrodynamic dispersive models to define physically relevant quantities (e.g., potentials, energy, flow properties at an arbitrary depth, etc.). We also present solutions for the zero dispersion limit of conservation laws and demonstrate that the RD scheme applied to the proposed hyperbolic system is robust and can capture physical oscillations associated with the generated dispersive shocks.

\section{Hyperbolic Dispersion}

In this section, we start with a time-dependent dispersive PDE, and reformulate it to a first-order system that can be successfully transformed to a first-order hyperbolic system.

Consider the following linear dispersive PDE that is often referred to as the Airy equation,

$$
\partial_{t} u=\epsilon \partial_{x x x} u,
$$

where $\epsilon$ is the dispersion coefficient (positive or negative). Following the process we outlined in Ref. 21 (although other choices may also be possible), we consider here the semi-discrete form of Eq. (2) obtained with some implicit time integration scheme:

$$
\frac{\alpha}{\Delta t} u=\epsilon \partial_{x x x} u+s(x),
$$

where $\alpha$ and $s(x)$ depend on the time discretization and the known values of $u .^{1,21}$ We then replace the 
semi-discrete PDE by the steady limit of the pseudo-time dependent system

$$
\begin{aligned}
& \partial_{\tau} u=\epsilon \partial_{x} q-\frac{\alpha}{\Delta t} u+s(x), \\
& \partial_{\tau} p=\frac{1}{T_{\epsilon}}\left(\partial_{x} u-p-\gamma u\right), \\
& \partial_{\tau} q=\frac{1}{T_{\epsilon}}\left(\gamma \partial_{x} u+\partial_{x} p-q\right),
\end{aligned}
$$

where $\tau$ is the pseudo time, $t$ is the physical time, and $T_{\epsilon}$ and $\gamma$ are, respectively, the dispersion relaxation time and an arbitrary constant, both to be defined later.

It is easy to verify that $u$ in the above system satisfies the original semi-discrete dispersion equation at pseudo steady state (see also Refs. 21 and 1). Note that, the proposed system with $\gamma=0$ reduces to the first-order system formulation that is proven in Ref. 25 not to be hyperbolic. The study of the characteristic polynomial of the Jacobian matrix of the quasi-linear differential operator on the right hand side of Eq. (3) reveals that the form of $\gamma \neq 0$ is critical in constructing a hyperbolic system. For example, we have found that the following form of $\gamma$ could result in a hyperbolic formulation of the first-order system for dispersion

$$
\gamma=\beta+\frac{1}{\epsilon \beta^{2} T_{\epsilon}}
$$

where for dimensional consistency $\beta=\kappa / L_{\epsilon}, \kappa$ is an arbitrary constant, and $L_{\epsilon}$ is the dispersion length scale, to be defined later. With this choice of $\gamma$, the first-order system (3) admits the characteristic speeds

$$
\lambda_{1}^{\mathrm{disp}}=\frac{1}{\beta T_{\epsilon}}, \quad \lambda_{2}^{\mathrm{disp}}=\frac{\lambda_{1}^{\mathrm{disp}}}{2}\left(-1+\sqrt{1+4 \epsilon \beta^{3} T_{\epsilon}}\right), \quad \lambda_{3}^{\mathrm{disp}}=\frac{\lambda_{1}^{\mathrm{disp}}}{2}\left(-1-\sqrt{1+4 \epsilon \beta^{3} T_{\epsilon}}\right)
$$

with the following corresponding eigenvectors

$$
\mathbf{R}=\left[\begin{array}{ccc}
-\epsilon & -\lambda_{2}^{\text {disp }} T_{\epsilon} & -\lambda_{3}^{\text {disp }} T_{\epsilon} \\
\epsilon \beta & 1 & 1 \\
\lambda_{1}^{\text {disp }} & \beta-\lambda_{2}^{\text {disp }} /(\epsilon \beta) & \beta-\lambda_{3}^{\text {disp }} /(\epsilon \beta)
\end{array}\right]
$$

which is non-singular (unless $\kappa=-2,-\frac{1}{2}$, or 1 ).

We now define the relaxation time $T_{\epsilon}$ as the ratio between the dispersion length scale, $L_{\epsilon}$, and either of the $\lambda_{2}$ or the $\lambda_{3}$, and arrive at the following relation:

$$
T_{\epsilon}=\frac{L_{\epsilon}^{3}}{\epsilon}
$$

where we have chosen $\kappa=\kappa_{g} \equiv(1+\sqrt{5}) / 2$, which is denoted here as the golden ratio, satisfying $\kappa_{g}^{2}-\kappa_{g}-1=0$ by definition. We remark that the proposed system have three real eigenvalues with linearly independent eigenvectors for any positive $\kappa$ (except for $\kappa=1$, which makes the matrix of eigenvectors singular). Thus, the proposed first order system is hyperbolic. With the choice of the golden ratio, we arrive at $\gamma=2 / L_{\epsilon}$.

With the definitions of $T_{\epsilon}, \kappa\left(=\kappa_{g}\right), \beta$, and $\gamma$, we recast the proposed hyperbolic dispersion system in a vector form as

$$
\partial_{\tau} \mathbf{U}+\mathbf{A} \partial_{x} \mathbf{U}=\mathbf{Q}
$$

where

$$
\mathbf{U}=\left[\begin{array}{c}
u \\
p \\
q
\end{array}\right], \quad \mathbf{A}=\mathbf{A}^{\mathrm{disp}}=\left[\begin{array}{ccc}
0 & 0 & -\epsilon \\
-1 / T_{\epsilon} & 0 & 0 \\
-\gamma / T_{\epsilon} & -1 / T_{\epsilon} & 0
\end{array}\right], \quad \mathbf{Q}=\left[\begin{array}{c}
-\frac{\alpha}{\Delta t} u+s(x) \\
-(p+\gamma u) / T_{\epsilon} \\
-q / T_{\epsilon}
\end{array}\right]
$$

The three real eigenvalues of the Jacobian matrix $\mathbf{A}^{\text {disp }}$ can be shown to be (see also Fig. 1)

$$
\lambda_{1}^{\mathrm{disp}}=\frac{L_{\epsilon}}{\kappa_{g} T_{\epsilon}}, \quad \lambda_{2}^{\mathrm{disp}}=\kappa_{g} \lambda_{1}^{\mathrm{disp}}, \quad \lambda_{3}^{\mathrm{disp}}=-\kappa_{g}^{2} \lambda_{1}^{\mathrm{disp}},
$$

and the corresponding right and left eigenvectors are 


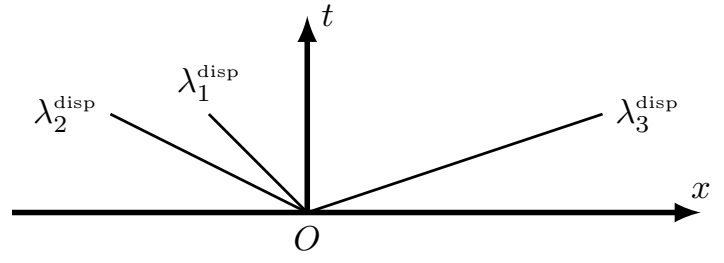

(a) $\epsilon<0$

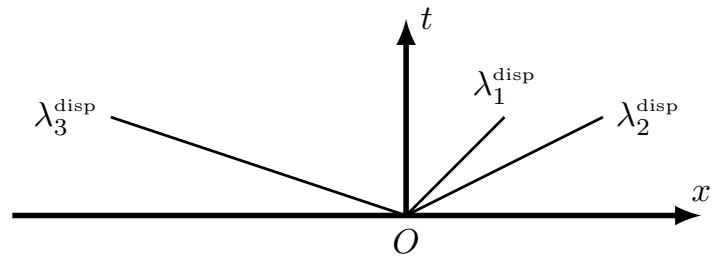

(b) $\epsilon>0$

Figure 1: Schematics of the dispersion waves structures for the proposed hyperbolic dispersion system.

$$
\mathbf{R}=\left[\begin{array}{ccc}
-\kappa_{g} L_{\epsilon}^{2} & -L_{\epsilon}^{2} & \kappa_{g} L_{\epsilon}^{2} \\
L_{\epsilon} \kappa_{g}^{2} & L_{\epsilon} & L_{\epsilon} \\
1 & 1 & \kappa_{g}^{2}
\end{array}\right], \quad \mathbf{L}=\frac{1}{\kappa_{g}^{2}+1}\left[\begin{array}{ccc}
1 / L_{\epsilon}^{2} & \kappa_{g}^{2} / L_{\epsilon} & -\kappa_{g} \\
-\left(\kappa_{g}^{2}+1\right) / L_{\epsilon}^{2} & -\left(\kappa_{g}^{2}+1\right) / L_{\epsilon} & \kappa_{g}^{2}+1 \\
1 / L_{\epsilon}^{2} & 1 /\left(\kappa_{g}^{2} L_{\epsilon}\right) & 1 / \kappa_{g}
\end{array}\right]
$$

We now define the dispersion length scale, $L_{\epsilon}$, by first inserting the Fourier mode of phase angle $\theta \in[0, \pi]$ (i.e., $\mathbf{U}=\mathbf{U}_{0} e^{I \theta / h}$ ), where $\mathbf{U}_{0}$ is a constant vector, $h$ is a grid spacing, and $I=\sqrt{-1}$, into the hyperbolic dispersion system. We then evaluate the eigenvalues of the Fourier-transformed operator with the $\kappa_{g}$ value, and expand them for small $\theta$ to obtain the leading terms:

$$
\lambda_{1}=-\frac{I \epsilon}{h^{3}} \theta^{3}+\frac{2 I \epsilon L_{\epsilon}^{2}}{h^{5}} \theta^{5}+\frac{2 \epsilon L_{\epsilon}^{3}}{h^{6}} \theta^{6}+O\left(\theta^{7}\right), \quad \lambda_{2 / 3}=-\frac{\epsilon}{L_{\epsilon}^{3}} \pm \frac{I \sqrt{2} \epsilon}{L_{\epsilon}^{2} h} \theta+O\left(\theta^{2}\right) .
$$

The first eigenvalue is clearly an approximation to the Fourier symbol of the operator $\epsilon \partial_{x x x} u$, with a secondorder error in terms of $\theta$. The other two represent damping modes with the real part, $-\epsilon / L_{\epsilon}^{3}$, but also have propagation in the next leading term, $\pm I \sqrt{2} \epsilon \theta /\left(L_{\epsilon}^{2} h\right)$. To determine the dispersion length scale and possibly enhance the effects of error propagation during transient pseudo-time, we equate the imaginary parts of the second and the third eigenvalues with the leading term of the first eigenvalue (i.e., $\frac{\epsilon}{h^{3}} \theta^{3}=\frac{\sqrt{2} \epsilon}{L_{\epsilon}^{2} h} \theta$ ) for the smoothest mode (i.e., lowest frequency error mode) by setting $\theta=\pi h$. After some algebra, we arrive at the following dispersion length scale:

$$
L_{\epsilon}= \pm \frac{2^{\frac{1}{4}}}{\pi}
$$

where the positive and negative length scales are associated with the positive and negative dispersion coefficient, $\epsilon$, respectively. For all the numerical examples presented in Sec. VI, we observed experimentally that both doubling and halving the above dispersion length scale result in a larger number of linear relaxations and therefore, the above dispersion length scale appears to be optimum.

The proposed hyperbolic dispersion system is now completely defined. In the next section, we present an extension of this system to an advective-dispersive PDE (such as KdV) and show that a fully-hyperbolic system formulation of such PDEs can also be found. The general extension of the proposed hyperbolic system for a general advection-diffusion-dispersion equation is discussed in Sec. IV.

\section{Hyperbolic Advection-Dispersion}

In this section, we briefly present an immediate extension of the hyperbolic dispersion system to general advective-dispersive PDEs (such as KdV). Here, we present one possible fully-hyperbolic advective-dispersive system (in one-dimension), which could be beneficial in imposing characteristic boundary conditions (BCs). The subject of the characteristic $\mathrm{BC}$ is, however, beyond the scope of this paper and therefore, the effects of the formulation on characteristic $\mathrm{BC}$ is left for future investigations.

Consider general advective-dispersive PDEs: $\partial_{t} u+\partial_{x} f=\epsilon \partial_{x x x} u$, where advection speed is defined as 
$a(u)=\partial f / \partial x$. One possible hyperbolic formulation for advective-dispersive PDEs is:

$$
\begin{aligned}
& \partial_{\tau} u=-a \partial_{x} u+\epsilon \partial_{x} q-\frac{\alpha}{\Delta t} u+s(x), \\
& \partial_{\tau} p=\left(\frac{1}{T_{a}}+\frac{1}{T_{\epsilon}}\right)\left(\partial_{x} u-p-\gamma u\right), \\
& \partial_{\tau} q=\frac{1}{T_{\epsilon}}\left(\gamma \partial_{x} u+\partial_{x} p-q\right),
\end{aligned}
$$

where $T_{a}$ is the advection relaxation time, $\gamma=\beta+\frac{T_{\epsilon}}{\epsilon T^{2} \beta^{2}}-\frac{a}{\epsilon \beta} \frac{T_{\epsilon}}{T}$, and $\frac{1}{T}=\frac{1}{T_{a}}+\frac{1}{T_{\epsilon}}$. This system has the following three characteristic speeds

$$
\lambda_{1}^{\text {adv-disp }}=\frac{1}{\beta}\left(\frac{1}{T_{a}}+\frac{1}{T_{\epsilon}}\right), \lambda_{2,3}^{\text {adv-disp }}=\frac{1}{2}\left(a-\lambda_{1}^{\text {adv-disp }} \pm \sqrt{\left(a-\lambda_{1}^{\text {adv-disp }}\right)^{2}+\frac{4 \epsilon \beta}{T_{\epsilon}}}\right),
$$

with the corresponding right eigenvectors expressed as

$$
\mathbf{R}=\left[\begin{array}{ccc}
-\epsilon & -\lambda_{2}^{\text {adv-disp }} T & -\lambda_{3}^{\text {adv-disp }} T \\
\epsilon \beta & 1 & 1 \\
\lambda_{1}^{\text {adv-disp }}-a & \beta T / T_{\epsilon}-\lambda_{2}^{\text {adv-disp }} /(\epsilon \beta) & \beta T / T_{\epsilon}-\lambda_{3}^{\text {adv-disp }} /(\epsilon \beta)
\end{array}\right]
$$

We follow the same procedure as given in Sec. II, and obtain the relaxation time as $T=-\left(a L T_{\epsilon}-\right.$ $\left.\sqrt{a^{2} L^{2} T_{\epsilon}^{2}+4 L T_{\epsilon}(\beta L+1)}\right) /(2 \epsilon \beta)$. With this relaxation time, we can show that the eigenvalues are always real, and matrix of right eigenvectors is non-singular with $\kappa>0$ (except for $\kappa=1$ ). Thus, the presented system is fully-hyperbolic for $\kappa>0$ and $\kappa \neq 1$.

An optimum length scale $L$ can be obtained by a Fourier analysis, as discussed in Sec. II. However, this computation is not performed here, because for general numerical approximation we use the hyperbolic structure of advection, diffusion, and dispersion operators separately (see Sec. IV). The individual treatment of these operators makes the extension to multi-dimensions and/or more complex equations straightforward.

\section{Generalization: Hyperbolic Advection-Diffusion-Dispersion}

Here, we present an extension of the proposed hyperbolic dispersion formulation to a general hyperbolic advection-diffusion-dispersion system. This extension is not trivial and therefore, is reported here for completeness.

Consider a nonlinear advection-diffusion-dispersion equation

$$
\partial_{t} u+\partial_{x}(f)=\partial_{x}\left(\nu \partial_{x} u\right)+\epsilon \partial_{x x x} u,
$$

where $f$ is a nonlinear function of $u$, and the diffusion coefficient, $\nu$, may be a function of the solution variable $u$, and the advection speed is defined as $a(u)=\partial f / \partial u$. Using the hyperbolic dispersion system introduced in Sec. II, we propose the following hyperbolic system for a general advection-diffusion-dispersion equation, Eq. (17),

$$
\begin{aligned}
\partial_{\tau} u & =-\partial_{x} f+\partial_{x}(\nu p+\gamma \nu u)+\epsilon \partial_{x} q-\frac{\alpha}{\Delta t} u+s(x), \\
\partial_{\tau} p & =\left(\frac{1}{T_{\nu}}+\frac{1}{T_{\epsilon}}\right)\left(\partial_{x} u-p-\gamma u\right), \\
\partial_{\tau} q & =\frac{1}{T_{\epsilon}}\left(\gamma \partial_{x} u+\partial_{x} p-q\right),
\end{aligned}
$$

which is formulated such that it properly reduces to a pure hyperbolic advection-dispersion and a pure hyperbolic advection-diffusion system, respectively, in the dispersion limit $(\nu \rightarrow 0)$ and the diffusion limit $(\epsilon \rightarrow 0)$. 
Writing in vector form, Eq. (8), and following the non-unified approach of Ref. 23 for a separate treatment of advection, diffusion, and (in the this case) dispersion components, the flux Jacobian matrix $\mathbf{A}$ of the proposed hyperbolic advection-diffusion-dispersion formulation becomes

$$
\mathbf{A}=\mathbf{A}^{\text {adv }}+\mathbf{A}^{\text {diff }}+\mathbf{A}^{\text {disp }}=\left[\begin{array}{ccc}
a-\gamma \nu & 0 & 0 \\
0 & 0 & 0 \\
0 & 0 & 0
\end{array}\right]+\left[\begin{array}{ccc}
0 & -\nu & 0 \\
-1 / T_{\nu} & 0 & 0 \\
0 & 0 & 0
\end{array}\right]+\mathbf{A}^{\text {disp }},
$$

where $\mathbf{A}^{\text {disp }}$ is given in Eq. (9). Note that for simplicity in the discussion, we presented the flux Jacobian for a linear $\nu$, but the proposed first-order system is also applicable for a nonlinear $\nu$. Note also that we have included the flux Jacobian resulting from the presence of the $\gamma \nu u_{x}$ term in the first equation into the advective flux Jacobian, and not to the diffusion flux Jacobian; that is, we have treated $\gamma \nu$ as an added advection speed, which acts as an artificial advection in the diffusion limit (as $\gamma$ does not vanish when $\epsilon \rightarrow 0$ ). With the above formulation, we also recover the identical hyperbolic diffusion system (which is in fact a $[2 \times 2]$ system) of Ref. 20 and thus, the same eigenvalues, $\lambda_{1}^{\text {diff }}=-\sqrt{\nu / T_{\nu}}, \lambda_{2}^{\text {diff }}=\sqrt{\nu / T_{\nu}}$, the diffusion relaxation time, $T_{\nu}=L_{\nu}^{2} / \nu$, and the diffusion length scale, $L_{\nu}=1 / 2 \pi$, given in Ref. ?, are applicable here.

\section{Discretization and Implicit Solver}

The proposed hyperbolic advection-diffusion-dispersion system can now be discretized with a desired scheme, such as finite volume (FV), Discontinuous-Galerkin (DG), RD, etc. Here we describe the discretization within the RD framework.

Consider a one-dimensional domain discretized with $N$ randomly distributed nodes. We store solution vector $\mathbf{U}$ at each node denoted by $x_{j}$, where $j=1,2,3, \ldots, N$, and compute the cell residual, $\boldsymbol{\Phi}^{E}$, by integrating the right-hand-side of Eq. (8) over the cell defined by the node $j$ and $j+1$ (Fig. 2):

$$
\mathbf{\Phi}^{E}=\int_{j}^{j+1}\left(-\mathbf{A} \partial_{x} \mathbf{U}+\mathbf{Q}\right) d x
$$

where we follow the technique of Ref. 1 to compute cell residuals of both the steady and unsteady source terms.

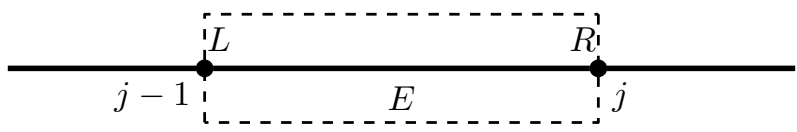

Figure 2: Schematic of the cell definition, and the left and the right nodes on each computational cell.

We now distribute the cell residual $\boldsymbol{\Phi}^{E}$ to the right and the left nodes of each cell with the following distribution function, which is obtained such that we can guarantee conservation when advection, diffusion, and dispersion are treated separately:

$$
\boldsymbol{\Phi}^{\mathrm{L}}=\mathbf{B}^{-} \boldsymbol{\Phi}^{\mathbf{E}}, \quad \boldsymbol{\Phi}^{\mathrm{R}}=\mathbf{B}^{+} \boldsymbol{\Phi}^{\mathbf{E}}, \quad \mathbf{B}^{ \pm}=\frac{1}{2} \mathbf{I} \pm \mathbf{D}^{\text {adv }} \pm \mathbf{D}^{\text {diff }} \pm \mathbf{D}^{\mathrm{disp}},
$$

where the positive and the negative signs of the advection, $\mathbf{D}^{\text {adv }}$, the diffusion, $\mathbf{D}^{\text {diff }}$, and the dispersion, $\mathbf{D}^{\text {disp }}$, stabilization terms correspond to the distributions of the cell residual $\mathbf{\Phi}^{E}$ to the right and the left nodes, respectively (see Fig. 2). We define these stabilization terms independently by reformulating the advection, the diffusion, and the dispersion projection matrices in the form of the above distribution function. To do this, we recall that an upwind distribution matrix can be constructed $\mathrm{as}^{20}$ (after some algebra):

$$
\mathbf{B}^{ \pm}=\frac{1}{2} \mathbf{R}\left[\begin{array}{ccc}
1 \pm \frac{\lambda_{1}}{\left|\lambda_{1}\right|} & 0 & 0 \\
0 & 1 \pm \frac{\lambda_{2}}{\left|\lambda_{2}\right|} & 0 \\
0 & 0 & 1 \pm \frac{\lambda_{3}}{\left|\lambda_{3}\right|}
\end{array}\right] \mathbf{L}=\frac{1}{2} \mathbf{I} \pm \frac{1}{2} \mathbf{A}\left(\mathbf{R}|\boldsymbol{\Lambda}|^{-1} \mathbf{L}\right)=\frac{1}{2} \mathbf{I} \pm \frac{1}{2} \mathbf{A} \sum_{l=1}^{3} \frac{1}{\left|\lambda_{l}\right|} \mathbf{\Pi}_{l},
$$

which results in the following advection, diffusion, and dispersion stabilization terms:

$$
\left.\mathbf{D}^{\text {adv }}=\frac{1}{2} \mathbf{A}^{\text {adv }} /(\mid a-\gamma \nu) \mid+\tilde{\epsilon}\right), \quad \mathbf{D}^{\text {diff }}=\frac{1}{2} \mathbf{A}^{\text {diff }} \sum_{l=1}^{2} \frac{1}{\left|\lambda_{l}^{\text {diff }}\right|} \mathbf{\Pi}_{l}^{\text {diff }}, \quad \mathbf{D}^{\text {disp }}=\frac{1}{2} \mathbf{A}^{\text {disp }} \sum_{l=1}^{3} \frac{1}{\left|\lambda_{l}^{\text {disp }}\right|} \mathbf{\Pi}_{l}^{\text {disp }}
$$


where $\tilde{\epsilon} \ll 1$ is added to avoid division by zero when the total advection speed, $a-\gamma \nu$, is identically zero. Note that the distribution matrix sums up to the identity matrix for each cell and therefore, we have conservation. The diffusion and dispersion projection matrices (i.e., $\boldsymbol{\Pi}^{\text {diff }}$ and $\boldsymbol{\Pi}^{\text {disp }}$ ), which are given here for convenience, are easily defined by projecting the diffusion and dispersion fluxes Jacobians onto their corresponding running waves (i.e., $\mathbf{A}^{\text {diff. }}=\sum_{l=1}^{2} \lambda_{l}^{\text {diff }} \boldsymbol{\Pi}_{l}^{\text {diff }}$, and $\mathbf{A}^{\text {disp. }}=\sum_{l=1}^{3} \lambda_{l}^{\text {disp }} \boldsymbol{\Pi}_{l}^{\text {disp }}$ ), to arrive at

$$
\begin{gathered}
\boldsymbol{\Pi}_{1}^{\text {diff }}=\frac{1}{\lambda_{1}^{\text {diff }}-\lambda_{2}^{\text {diff }}}\left[\begin{array}{ccc}
\lambda_{1}^{\text {diff }} & -\nu & 0 \\
-1 / T_{\nu} & -\lambda_{2}^{\text {diff }} & 0 \\
0 & 0 & 0
\end{array}\right], \quad \mathbf{\Pi}_{2}^{\text {diff }}=\mathbf{I}-\mathbf{\Pi}_{1}^{\text {diff }}, \\
\boldsymbol{\Pi}_{1}^{\text {disp }}=\frac{1}{\kappa_{g}^{2}+1}\left[\begin{array}{ccc}
-\kappa_{g} & -\kappa_{g}^{3} L_{\epsilon} & \kappa_{g}^{2} L_{\epsilon}^{2} \\
\frac{\kappa_{g}^{2}}{L_{\epsilon}} & \kappa_{g}^{4} & -\kappa_{g}^{3} L_{\epsilon} \\
\frac{1}{L_{\epsilon}^{2}} & \frac{\kappa_{g}^{2}}{L_{\epsilon}} & -\kappa_{g}
\end{array}\right], \mathbf{\Pi}_{2}^{\text {disp }}=\left[\begin{array}{ccc}
1 & L_{\epsilon} & -L_{\epsilon}^{2} \\
-\frac{1}{L_{\epsilon}} & -1 & L_{\epsilon} \\
-\frac{1}{L_{\epsilon}^{2}} & -\frac{1}{L_{\epsilon}} & 1
\end{array}\right], \boldsymbol{\Pi}_{3}^{\text {disp }}=\mathbf{I}-\boldsymbol{\Pi}_{1}^{\text {disp }}-\mathbf{\Pi}_{2}^{\text {disp }},
\end{gathered}
$$

where $\mathbf{I}$ is an identity matrix.

We now follow the procedure described in Ref. 1, and construct an implicit solver with a second-order compact Jacobian matrix, which we compute numerically with an automatic-differentiation technique based on an operator-overloading algorithm.

\section{Results}

In this section, we examine and verify the accuracy of the proposed hyperbolic advection-diffusiondispersion system. The presented examples are solved by discretizing the hyperbolic system using the RD scheme discussed in Sec. V. We relax the linearized system with a Gauss-Seidel (GS) algorithm and reduce the linear residuals by three orders of magnitude with the maximum of 1000 relaxations. This typically takes, on average, about 40-50 GS relaxations with an under relaxation parameter of 0.8. The implicit solver is then continued until ten orders of magnitude reduction is achieved for all the equations; this usually takes about 4-5 Newton iterations (per time step).

We first present an example for solving a nonlinear advection-diffusion-dispersion equation through the method-of-manufactured solution. This example is used to verify the order of accuracy of the scheme. We then present two more examples; one for solving the classical KdV equation with small dispersion coefficient, and one to demonstrate the capability of the proposed scheme in resolving physical oscillations in the zero dispersion limit.

\section{A. Example 1: Order of accuracy verification}

Consider the following nonlinear advection-diffusion-dispersion equation:

$$
\partial_{t} u+\partial_{x} f=\partial_{x}\left(\nu \partial_{x} u\right)+\epsilon \partial_{x x x} u+\tilde{s}(x, u)
$$

where $f=3 u^{2}, \nu=0.5, \epsilon=1$ and $\tilde{s}$ is the manufactured source term. We seek, through the method of manufactured solution, a time-dependent two-soliton solution of the (generalized) KdV equation: ${ }^{26}$

$$
u^{e}(x, t)=\frac{\left(\eta_{2}-\eta_{1}\right)\left(\eta_{1} \operatorname{sech}^{2}\left[\chi\left(\eta_{1}\right)\right]+\eta_{2} \operatorname{csch}^{2}\left[\chi\left(\eta_{2}\right)\right]\right)}{\left(\sqrt{\eta_{1}} \tanh \left[\chi\left(\eta_{1}\right)\right]-\sqrt{\eta_{2}} \operatorname{coth}\left[\chi\left(\eta_{2}\right)\right]\right)^{2}}
$$

where

$$
\chi(\eta)=\sqrt{\frac{\eta}{2}}(x-2 \eta t-\tilde{a}),
$$

and $\eta_{1}>0, \eta_{2}>0$, and $\tilde{a}$ are arbitrary constants. Here we consider $\eta_{1}=0.5, \eta_{2}=1.0$, and $\tilde{a}=0.1$, but similar results are also obtained with other values.

After reformulating Eq. (28) in the form of the proposed hyperbolic advection-diffusion-dispersion system discussed in Sec. IV, we apply the efficient fourth-order RD scheme of Ref. 1 for the spatial discretization, along with the A-stable second-order Backward-Differencing-Formula (BDF2) for the temporal discretization 
to the hyperbolic system. We then solve the system of equations with an imposed Dirichlet boundary condition (i.e., the time-depended solutions at the boundary nodes are fixed) and $u^{e}(x, 0)$ as an initial condition.

Figure 3 shows the predicted solution $u$ and its first and second gradients (i.e., $u_{x}$ and $u_{x x}$ ) at different times. These solutions are obtained with $\Delta t=0.01$ using 60 randomly distributed grid points in $x \in(0,30)$. As shown, the predicted fourth-order results are in a very good agreement with the exact values even on

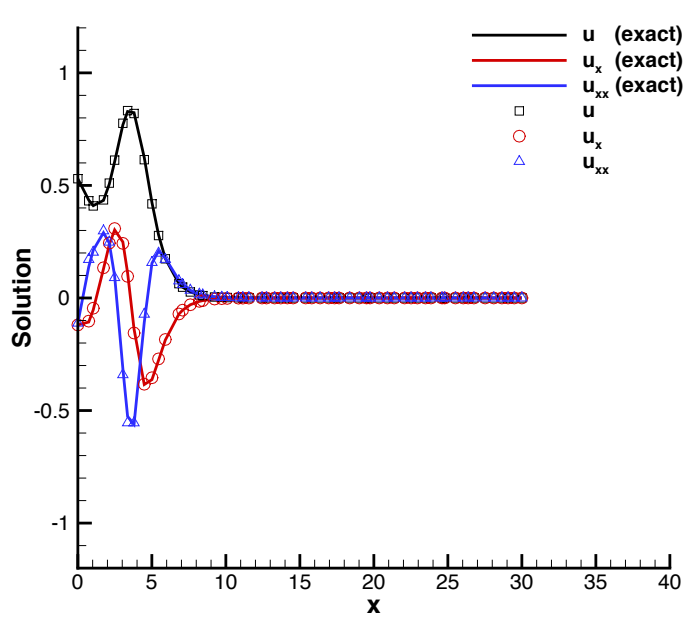

(a) $t=1.0$

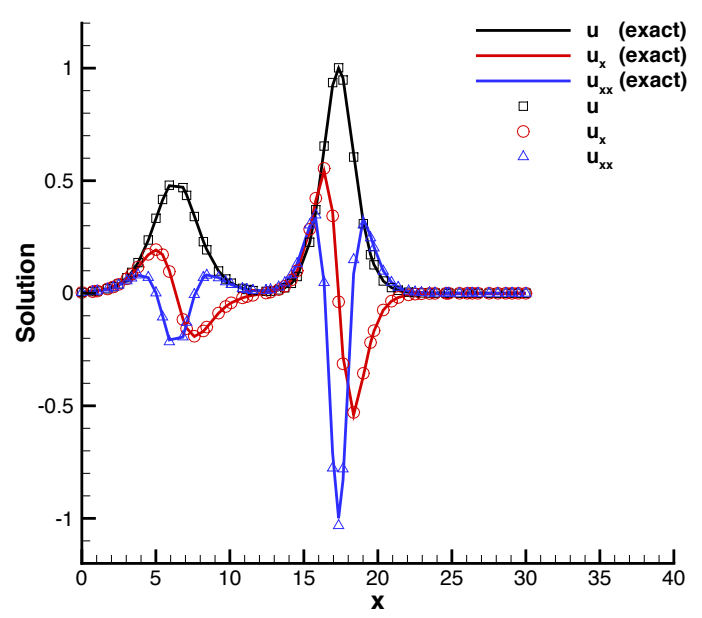

(c) $t=8.0$

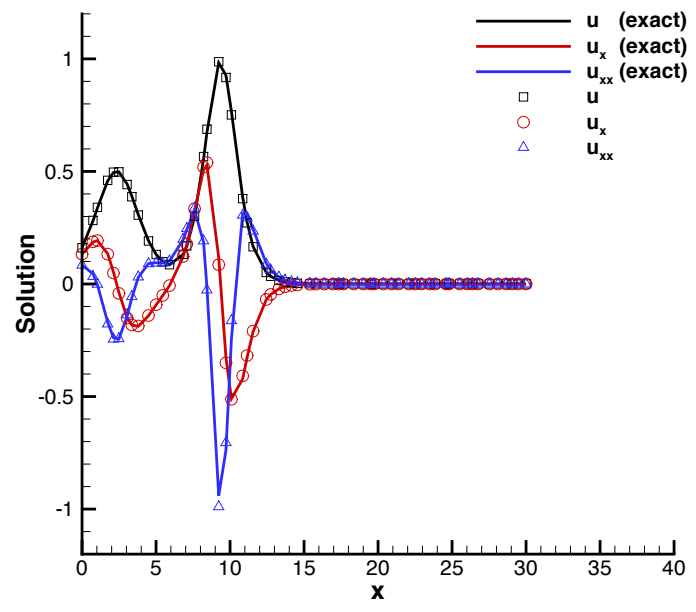

(b) $t=4.0$

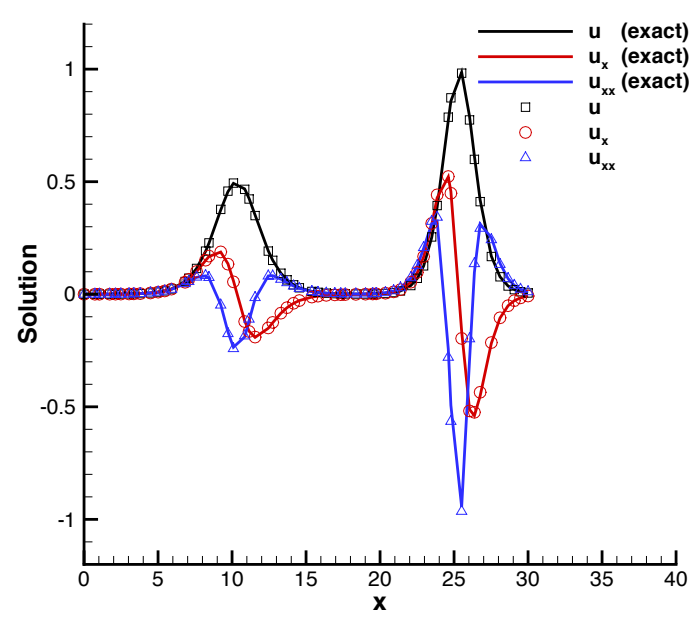

(d) $t=12.0$

Figure 3: Two-soliton KdV with method of manufactured solution: comparison with the predicted (fourthorder spatial plus BDF2) and exact solutions (including $u_{x}$ and $u_{x x}$ ) at different times obtained on a randomly distributed nodes $(N=60)$ in $x \in[0,30](\Delta t=0.01)$.

such a relatively coarse grid.

Figure 4 shows a comparison between the fourth-order solutions and the second-order solutions for the same problem on the same randomly distributed grid. The presented results correspond to the solution at $t=10$ with $\Delta t=0.01$. This figure shows that the second-order scheme over/under predicts the peaks and valleys of the gradients, while the fourth-order scheme captures them more accurately.

We also verified the formal temporal and spatial orders of accuracy for the solution, gradient, and the Hessian (second-derivative) on a series of grids with randomly distributed nodes. The error convergence is 


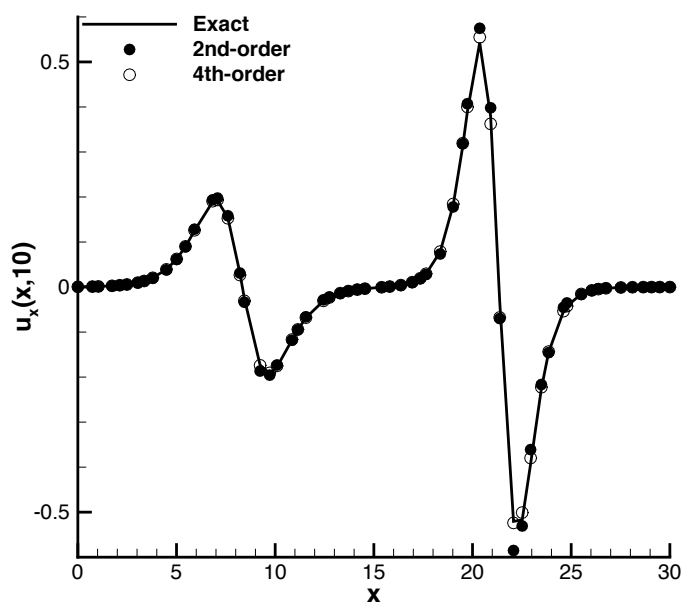

(a) $u_{x}$ at $t=10$

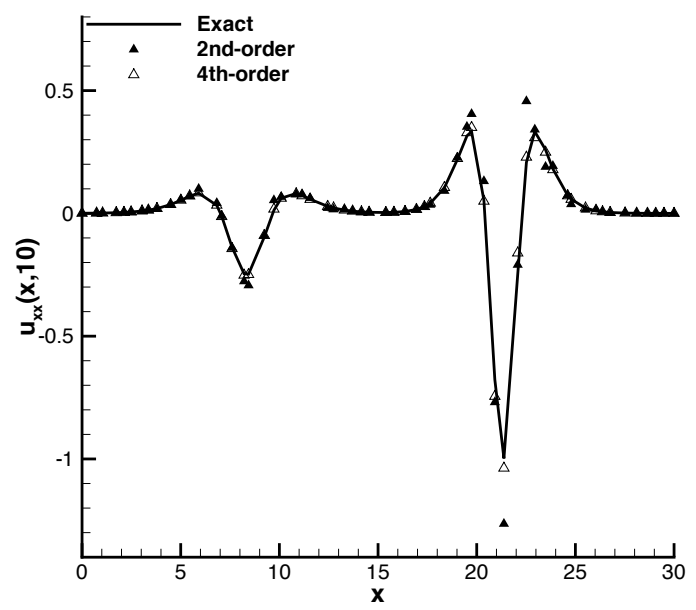

(b) $u_{x x}$ at $t=10$

Figure 4: Comparison between the predicted second- and fourth-order solution gradients for the two-soliton $\mathrm{KdV}$ problem computed on a randomly distributed nodes $(N=60)$ in $x \in[0,30](t=10, \Delta t=0.01)$.

obtained by computing the $L_{1}$ norms of the solutions (i.e., $\mathbf{L}_{1}=\sum_{j=1}^{N}\left|\mathbf{U}_{j}-\mathbf{U}_{j}^{e}\right| / N$, where $\mathbf{U}$ is the solution vector, the superscript $e$ denotes the exact value, and $N$ is the total number of grid points). Similar results are also obtained with the $L_{2}$ norm and therefore not shown. For temporal accuracy verification, we used a grid with 640 randomly distributed points, and varied $\Delta t$. For spatial accuracy, we fixed $\Delta t=0.001$ and used a series of randomly distributed grids. These results are shown in Fig. 5, indicating that all solution variables, including the first two solution gradients (i.e., $u_{x}$ and $u_{x x}$ ) are fourth-order accurate in space and second-order accurate in time. This is significant because a sixth-order accurate solution is typically needed with conventional schemes to achieve fourth-order accurate second-derivatives. The reconstructed solution gradients, which are computed using a cubic polynomial fit of the fourth-order computed solution, are at least 1-2 orders of magnitude less accurate than the computed solution gradients. The orders of accuracy of the reconstructed solution gradients are also shown in Fig. 5 for comparison.

\section{B. Example 2: Colliding soliton waves}

Consider the following classical KdV equation:

$$
\partial_{t} u+\partial_{x} f=\epsilon \partial_{x x x} u
$$

where $f=u^{2} / 2$ and $\epsilon<0$. The following initial condition results in a time-dependent colliding two-soliton solution:

$$
u(x, 0)=3 \eta_{1} \operatorname{sech}^{2}\left(\frac{1}{2} \sqrt{-\eta_{1} / \epsilon}\left[\left(x-x_{1}\right)-\eta_{1} t\right]\right)+3 \eta_{2} \operatorname{sech}^{2}\left(\frac{1}{2} \sqrt{-\eta_{2} / \epsilon}\left[\left(x-x_{2}\right)-\eta_{2} t\right]\right)
$$

where $\eta_{1}$ and $\eta_{2}$ are arbitrary positive constants, and $x_{1}$ and $x_{2}$ are the initial locations of the two soliton waves. Following the example given in Ref. 27, we consider $\eta_{1}=0.3, \eta_{2}=0.1, x_{1}=0.4, x_{2}=0.8$ and $\epsilon=-4.84 \times 10^{-4}$ with a periodic boundary condition, and solve the classical KdV equation in $x \in[0,2]$ with 200 randomly distributed nodes. Similar results are also obtained with other values and therefore, not shown.

Figure 6 shows the predicted solution $u$ and its gradient and Hessian (i.e., $u_{x}$ and $u_{x x}$ ) at $t=1,2,3$. The initial solution is also shown as reference. These solutions are obtained with $\Delta t=0.01$ using the fourth-order (spatial) RD scheme (with BDF2 for time discretization) of Ref. 1. We note that these results are converged and further grid refinement does not change the results qualitatively. 


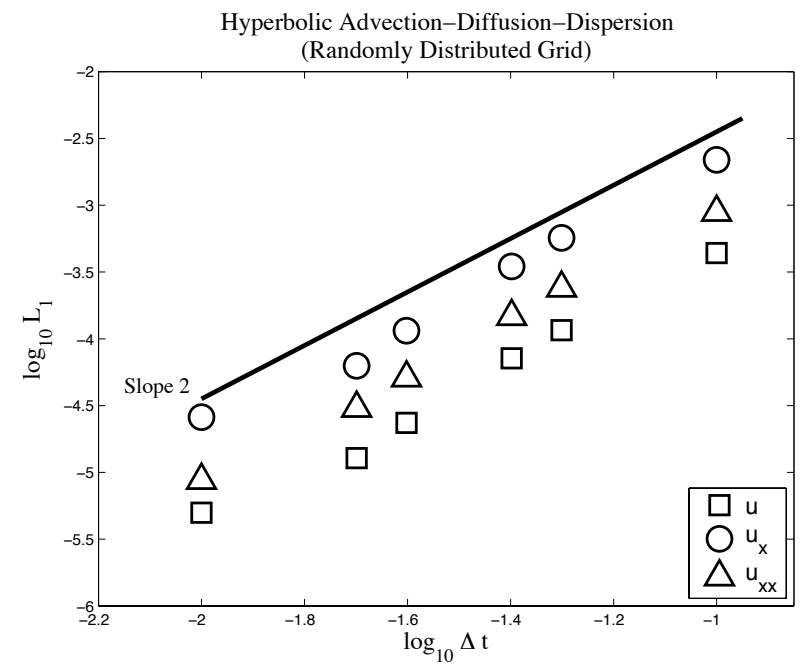

(a) Temporal accuracy (BDF2), $N=640$

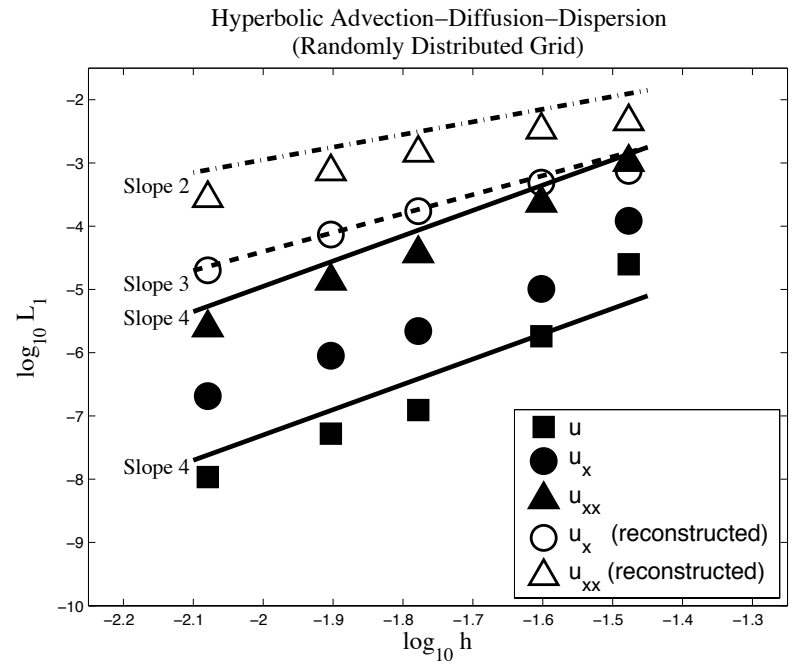

(b) Spatial accuracy (fourth-order), $\Delta t=0.001$

Figure 5: Temporal and spatial accuracy verifications of the proposed hyperbolic advection-diffusiondispersion system for the solution variable and its first two derivatives using the fourth-order RD-GT scheme (with BDF2 for temporal discretization) ${ }^{1}$ on randomly distributed nodes.

\section{Example 3: Dispersive shock}

Consider a dispersive nonlinear Burger equation (i.e., Eq. (31) when $\epsilon \rightarrow 0^{+}$). This equation has a dispersive behavior and produces continuous wavelets in the vicinity of the discontinuity (aka dispersive shock waves). In this example, we illustrate the capability of our code in resolving high-frequency wavelets for very small $\epsilon$. Figure 7 shows the predicted results for $\epsilon=10^{-3}$ and $10^{-4}$. The predicted high-order gradients and Hessians (second-derivatives) of the solution $u$ are also provided. The results show that physical oscillations are captured and solutions are noise-free particularly before and after the dispersive shocks (others reported solutions with some numerical oscillations, see e.g., Ref. 24). For comparison, the reconstructed gradient (third-order) and Hessian (second-order) are also shown for comparison. We note that these solutions are converged (i.e., no noticeable change in results are observed with further grid refinement) and we experienced no instability in obtaining these solutions.

\section{Conclusions}

We have introduced, for the first time, a first-order system approach for general advection-diffusiondispersion equations. We showed that the proposed system has real eigenvalues with linearly independent eigenvectors, and thus is hyperbolic. We apply the fourth-order RD scheme of Ref. 1 to the proposed hyperbolic system, and solved several time-dependent dispersive equations, including the classical two-soliton $\mathrm{KdV}$ and a dispersive shock case. We demonstrated that the predicted results, including the gradient and Hessian (i.e., $u_{x}$ and $u_{x x}$ ), are in a very good agreement with the exact solutions. We also showed that the $\mathrm{RD}$ scheme applied to the proposed system can capture dispersive shocks with no numerical oscillations. The design order of accuracy of the scheme (fourth-order spatial and second-order temporal) is also verified, and we achieved equal order of accuracy for the solution, the gradient, and the Hessian.

\section{Acknowledgments}

The first and last authors would like to thank the Center Chief Technology Office of NASA Langley Research Center for their support through the Center Innovation Fund (CIF) . 


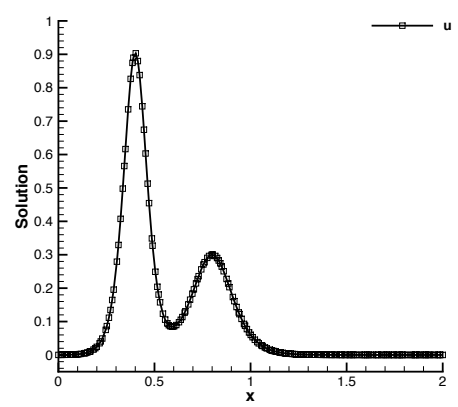

(a) $t=0, u$

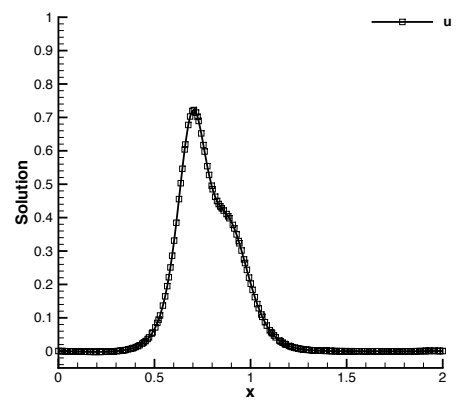

(d) $t=1, u$

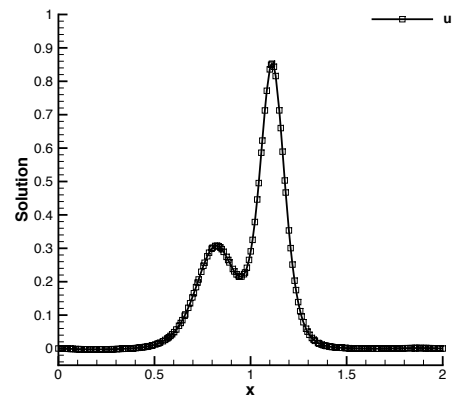

(g) $t=2, u$

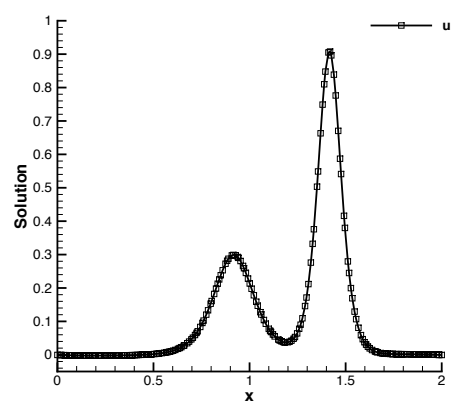

(j) $t=3, u$

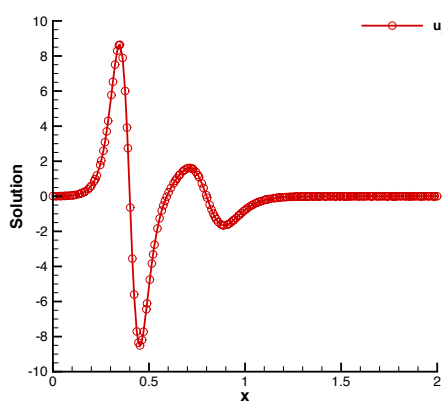

(b) $t=0, u_{x}$

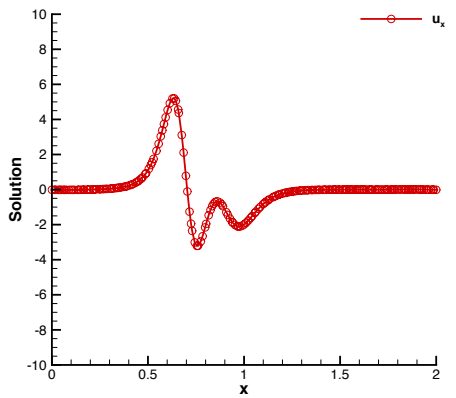

(e) $t=1, u_{x}$

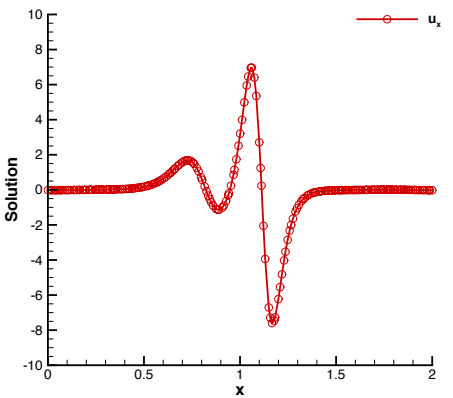

(h) $t=2, u_{x}$

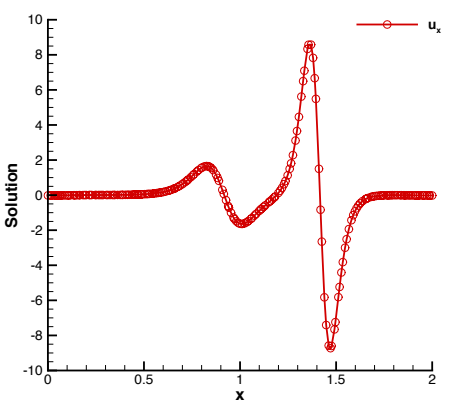

(k) $t=3, u_{x}$

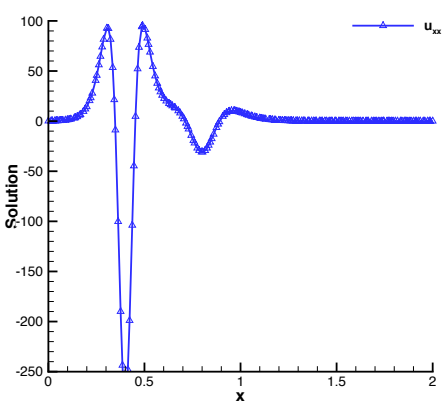

(c) $t=0, u_{x x}$

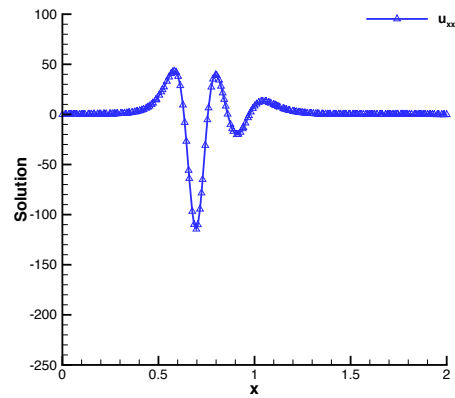

(f) $t=1, u_{x x}$

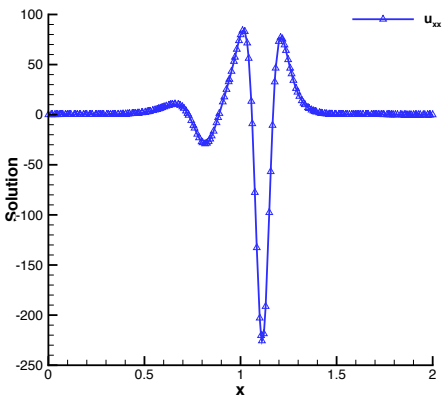

(i) $t=2, u_{x x}$

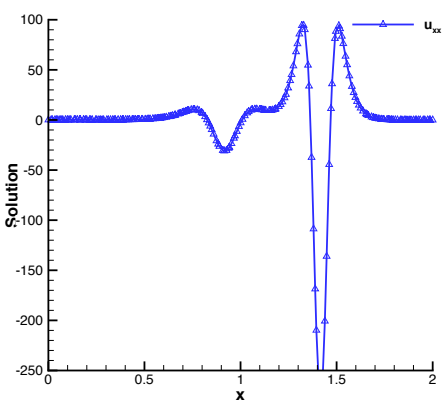

(l) $t=3, u_{x x}$

Figure 6: Two-soliton solution to $u_{t}+u u_{x}=\epsilon u_{x x x}$ : Predicted (fourth-order spatial plus BDF2) results with 200 randomly distributed grid points in a periodic domain $x \in[0,2]\left(\epsilon=-4.84 \times 10^{-4}, \Delta t=0.01\right)$. 


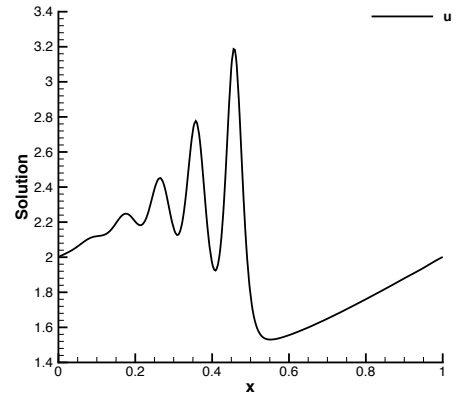

(a) $N=300, t=0.5, \epsilon=-10^{-4}$

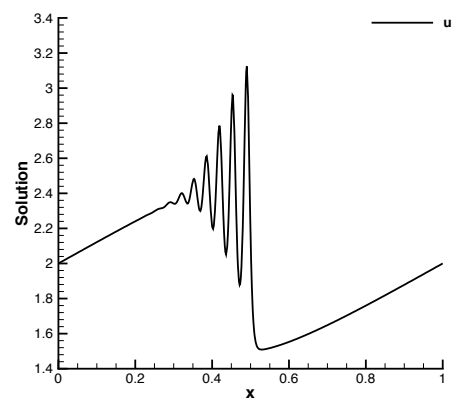

(d) $N=500, t=0.5, \epsilon=-10^{-5}$

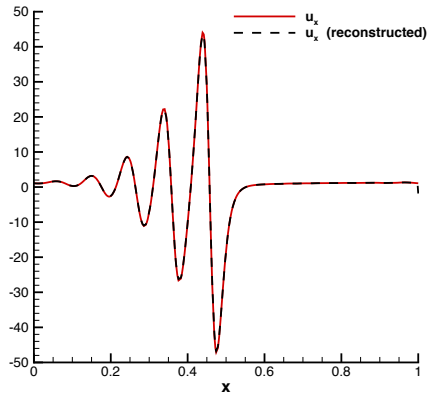

(b) $N=300, t=0.5, \epsilon=-10^{-4}$

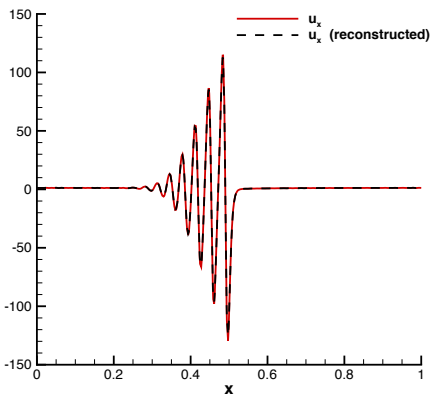

(e) $N=500, t=0.5, \epsilon=-10^{-5}$

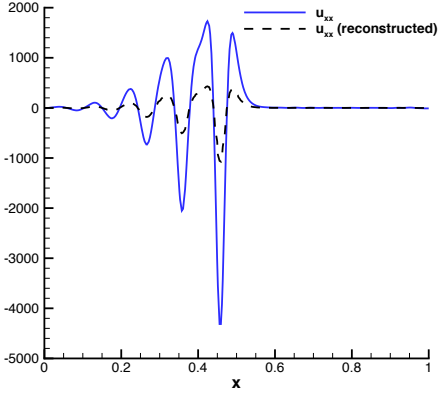

(c) $N=300, t=0.5, \epsilon=-10^{-4}$

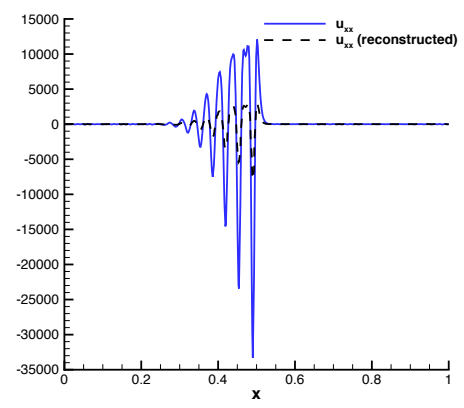

(f) $N=500, t=0.5, \epsilon=-10^{-5}$

Figure 7: Dispersive shock case (zero dispersion limit): $u_{t}+u u_{x}=\epsilon u_{x x x}, \epsilon \rightarrow 0, u(x, 0)=2+1 / 2 \sin (2 \pi x)$ : predicted solution, its gradient and Hessian (second-derivative) using the fourth-order (plus BDF2) RD scheme applied to the proposed hyperbolic dispersion system on randomly distributed grid points in a periodic domain $x \in[0,1]$. 


\section{References}

${ }^{1}$ A. Mazaheri and H. Nishikawa. Very efficient high-order hyperbolic schemes for time-dependent advection-diffusion problems: Third-, fourth-, and sixth-order. Computers and Fluids, 102:131-147, 2014.

${ }^{2} \mathrm{H}$. Leblond. Interaction of two solitary waves in a ferromagnet. J. Phys. A: Math. Gen., 28:3763, 1995.

${ }^{3}$ W. Wan, S. Jia, and W. Fleischer. Dispersive superfluid-like shock waves in nonlinear optics. Nature Physics, 3(1):46-51, 2007.

${ }^{4}$ N. Ghofraniha, C. Conti, G. Ruocco, and S. Trillo. Shocks in nonlocal media. Physical Review Letters, 99:043093, 2007.

${ }^{5}$ M.A. Hoefer, M.J. Ablowitz, I. Coddington, E.A. Cornell, P. Engels, and V. Schweikhard. Dispersive and classical shock waves in Bose-Einstein condensates and gas dynamics. Phys. Rev. A, 74:023623, 2006.

${ }^{6}$ M.A. Hoefer. Shock waves in dispersive Eulerian fluids. Journal of Nonlinear Science, 24(3):525-577, 2014.

${ }^{7}$ R.K. Smith, N. Crook, and G. Roff. The morning glory: an extraordinary atmospheric undular bore. Quart. J. R. Met. Soc., 108:937-956, 1982.

${ }^{8}$ J.W. Rottman and R. Grimshaw. Atmospheric internal solitary waves. In Roger Grimshaw, editor, Environmental Stratified Flows, volume 3 of Topics in Environmental Fluid Mechanics, pages 61-88. Springer US, 2002.

${ }^{9}$ S. Glimsdal, G.K. Pedersen, C.B. Harbitz, and F. Lovholt. Dispersion of tsunamis: does it really matter? Nat. Hazards Earth Syst. Sci., 13:1507-1526, 2013.

${ }^{10} \mathrm{H}$. Chanson. Current knowledge in hydraulic jumps and related phenomena. a survey of experimental results. European Journal of Mechanics - B/Fluids, 28(2):191 - 210, 2009.

${ }^{11}$ D.L. Wilkinson and M.L. Banner. Undular bores. 6th Australian Hydraulics and Fluid Mechanics Conference, Adelaide (Australia), December 1977.

${ }^{12}$ N.K. Lowman and M.A. Hoefer. Dispersive shock waves in viscously deformable media. J. Fluid Mech., 718:524-557, 2013.

${ }^{13}$ S. Benzoni-Gavage. Planar traveling waves in capillary fluids. Differential Integral Equations, 26(3-4):433-478, 2013.

${ }^{14}$ C.-Y. Kao, A. Kurganov, and Z. Qu. A fast explicit operator splitting method for modified Buckley-Leverett equations. J. Sci. Comput., doi:10.1007/s10915-014-9950-x, 2014.

${ }^{15}$ R. Carles, R. Danchin, and J.-C. Saut. Madelung, Gross-Pitaevskii and Korteweg. Nonlinearity, 25(10):2843-2873, 2012.

${ }^{16}$ C.-K. Lin and K.C. Wu. Hydrodynamic limits of the nonlinear Klein-Gordon equation. J. Math. Pures Appl., 98:328-345, 2012.

${ }^{17}$ H.-L. Liu and J. Yan. A local discontinuous Galerkin method for the Korteweg-de Vries equation with boundary effect. J. Comput. Phys., 215:197-218, 2006.

${ }^{18}$ D. Lannes. The Water Waves Problem: Mathematical Analysis and Asymptotics. American Mathematical Society, Providence, Rhode Island, 2013.

${ }^{19}$ A.G. Filippini, S. Bellec, M. Colin, and M. Ricchiuto. On the nonlinear behavior of Boussinesq type models: amplitudevelocity vs ampltitude-flux forms. Coastal Engng., doi:10.1016/j.coastaleng.2015.02.003.2015, 2015.

${ }^{20} \mathrm{H}$. Nishikawa. A first-order system approach for diffusion equation. I: Second order residual distribution schemes. $J$. Comput. Phys., 227:315-352, 2007.

${ }^{21}$ A. Mazaheri and H. Nishikawa. First-order hyperbolic system method for time-dependent advection-diffusion problems. Technical Report TM-2014-218175, NASA, March 2014.

${ }^{22} \mathrm{H}$. Nishikawa. Accuracy-preserving boundary flux quadrature for finite-volume discretization on unstructured grids. J. Comput. Phys., 281:518-555, 2015.

${ }^{23}$ A. Mazaheri and H. Nishikawa. Improved second-order hyperbolic residual-distribution scheme and its extension to third-order on arbitrary triangular grids. J. Comput. Phys., 300:455-491, 2015.

${ }^{24} \mathrm{~J}$. Yan and C.-W. Shu. A local discontinuous Galerkin method for KdV type equations. SIAM J. Numer. Anal., 40:769-791, 2002.

${ }^{25}$ E.F. Toro and G.I. Montecinos. Advection-diffusion-reaction equation: hyperbolization and high-order ADER discritizations. SIAM J. Sci. Comput., 36(5):A2423-A2457, 2014.

${ }^{26}$ K. Brauer. http://www.usf.uos.de/institut/mitarbeiter/brauer/solitons.html. Accessed: July 20, 2015.

${ }^{27}$ A. Debussche and J. Printems. Numerical simulation of the stochastic Kortewegde Vries equation. Physica D: Nonlinear Phenomena, 134:200-226, 1999. 